\title{
REVIEW \\ Modeling Mulberry Shoot Elongation Growth
}

\author{
Kuniaki FUKUI* \\ Insect Biotechnology and Sericology Department, National Institute of Agrobiological Sciences \\ (Tsukuba, Ibaraki 305-8634, Japan)
}

\begin{abstract}
A shoot elongation model was constructed for prediction of mulberry growth that tried to use the length as a growth index. In the model, the shoot elongation rate was represented as the product of growth activity (GA) and potential rate (PR) which were defined as growth activity of mulberry shoot tips and the potential rate of shoot elongation realized at the maximum GA under a given environmental condition, respectively. GA and PR were functions of temperature, photoperiod and/or shoot length. The model was applied to shoot length data of three cultivars grown in a wide area in Japan, and its parameters were determined. The shoot lengths calculated by the model were similar to the measured values and represented annual fluctuation well. The model also showed good estimation of data which were not used for determining parameters. Therefore, the model is practical and seemed to represent the response of mulberry vegetative growth to temperature and photoperiod well.
\end{abstract}

Disciplines: Agro-meteorology/Sericulture

Additional key words: photoperiod, prediction model, shoot length, temperature

\section{Introduction}

Mulberry (Morus spp.) is a tree crop and its leaf is fed to silkworms in sericulture. The tree's assimilatory organs are harvested during various vegetative growth stages. Since the harvest is a heavy stress for the mulberry tree, it is important to evaluate and predict the state of the growth in order to obtain a stable yield and design silkworm rearing. In the past, Sugasawa ${ }^{7}$, and Kato and Inoue $^{6}$ constructed prediction models for leaf or shoot yield, and shoot length was predicted by Suzuki and Kanaya $^{8}$. However, their models make predictions only on fixed dates and are effective in only limited areas. Therefore, it is necessary to establish a way to predict the state of mulberry growth at anytime and anywhere. In order to satisfy such needs, the author developed a prediction model of mulberry shoot length, which has been used as a mulberry growth indicator in mulberry cultivation. This model is reviewed in this paper.

For developing the model, at first, relationships between mulberry shoot elongation and environmental factors, i.e. temperature and photoperiod, were investigated in a phytotron under natural sunlight ${ }^{1-3}$. Each relationship was represented by mathematical equations and the model was constructed by integrating each equation ${ }^{4}$. Parameters of the model were determined by applying shoot length growth data from a wide area in Japan ${ }^{5}$. The cultivar used in the experiments was Shin-ichinose when a specific cultivar is not referred to.

\section{Relationship between shoot elongation and temperature}

The relationship between shoot elongation rate and temperature when mulberries planted in pots were grown at a variety of constant temperature conditions is shown in Fig. 1. The shoot elongation rate increased linearly with increasing temperature below $27^{\circ} \mathrm{C}$, and the rate stopped increasing above about $27^{\circ} \mathrm{C}$. When mulberry saplings in pots were grown at various fluctuating temperatures but at the same average temperature conditions, the rates were almost similar ${ }^{2}$. According to the above results, the relationship between temperature and shoot elongation rate

\footnotetext{
Present address:

${ }^{1}$ Division of Genome and Biodiversity Research, National Institute of Agrobiological Sciences (Tsukuba, Ibaraki 305-8602, Japan)

*Corresponding author: e-mail fukuik@affrc.go.jp

Received 29 March 2007; accepted 30 August 2007.
} 


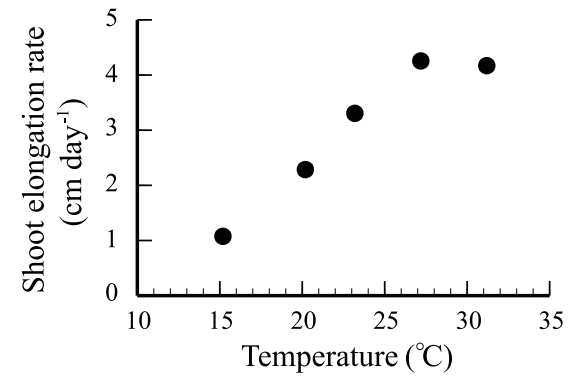

Fig. 1. Relationship between temperature and the shoot elongation rate of mulberry plants grown at five temperature conditions

was represented as follows. The rate is represented as a linear function of temperature until the rate reaches a maximum value, and after reaching the maximum value, the rate is assumed to be constant.

\section{Interactive effect of temperature and photoperiod on shoot elongation}

Potted mulberry plants were grown in phytotrons under natural sunlight, and their shoot growth response to temperature and photoperiod was investigated. Photoperiod was controlled by changing the growing seasons. The plants in the long photoperiod plot were grown from May to July, and the mulberries in the short photoperiod plot were grown from August to November.

A short photoperiod caused cessation of shoot growth (Fig. 2A), and this effect was prominent at $20^{\circ} \mathrm{C}$. On the contrary, temperatures higher than $24^{\circ} \mathrm{C}$ activated the growth activity for mulberry shoot tips. Opposite effects of short photoperiod and high temperature on the shoot growth might have brought about periodical increase and decrease of shoot elongation rate for individual mulberry plants (Fig. 2B).

To represent the interactive effect of temperature and photoperiod on shoot elongation, the shoot elongation rate was divided into two parts. One was the growth activity (GA) of mulberry shoot tips, and the other was potential rate $(\mathrm{PR})$ of shoot elongation. The shoot elongation rate is defined as the product of GA and PR. GA is described as dimensionless, ranging from 0 to 1 , and is a function of temperature and photoperiod. The value changes from that of the previous day. PR is defined as the potential rate of shoot elongation realized at the maximum GA under given environmental conditions. $\mathrm{PR}$ is described as a function of temperature. The relationship between shoot elongation rate and temperature given in the previous section is the same relationship between PR and temperature. The shape of functions of temperature and photoperiod in GA is assumed to be the same as the relationship between PR and temperature.

\section{Changes in shoot elongation rate with growth}

Potted mulberry plants were grown at constant temperature and photoperiod conditions. The shoot elongation rate increased sharply until about $50 \mathrm{~cm}$ shoot length (Fig. 3A). After that, the rate became constant. Shoot length at which the rate reached maximum values depended on growth temperature. The length was longer at higher temperature condition but the difference in the
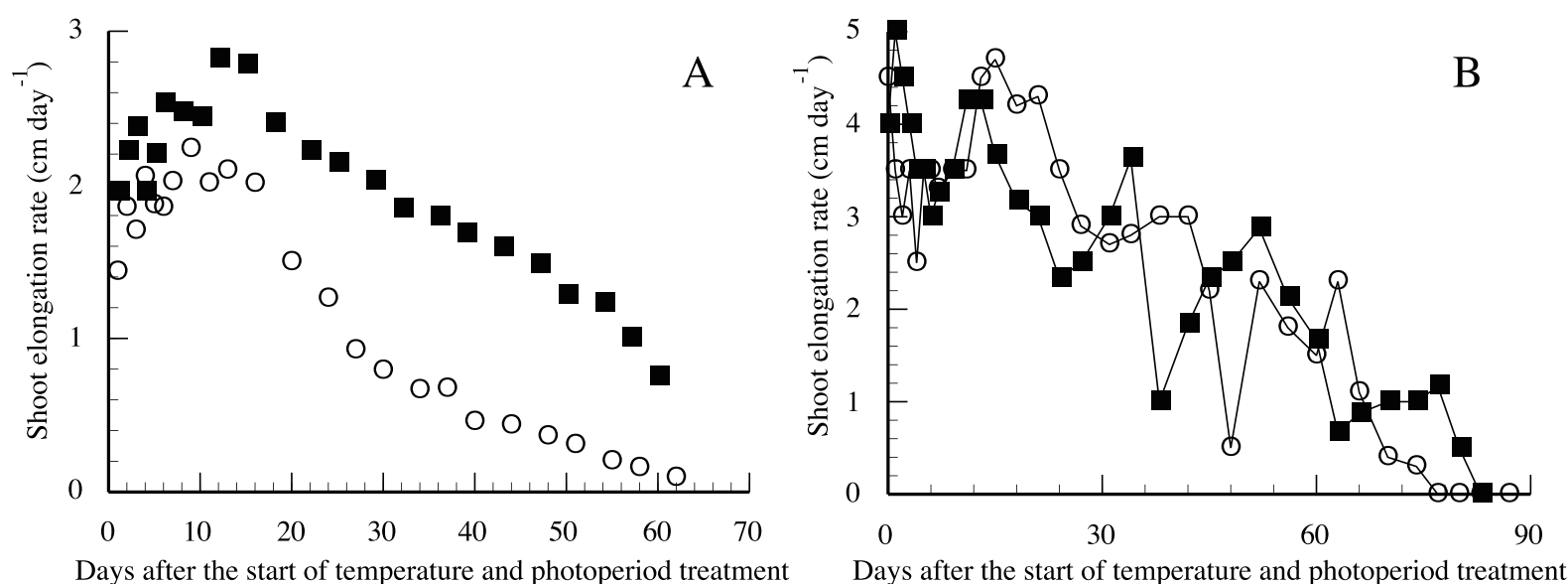

Days after the start of temperature and photoperiod treatment

Days after the start of temperature and photoperiod treatment

Fig. 2. Changes in the shoot elongation rate at different photoperiod and temperature conditions

A: Change in the shoot elongation rate at $20^{\circ} \mathrm{C}$ with long and short photoperiod conditions.

a: long photoperiod, O: short photoperiod.

B: Two examples of the change in shoot elongation rate of individual potted mulberry plants grown at $30^{\circ} \mathrm{C}$ with short photoperiod conditions. Each symbol shows individual mulberry plants. 

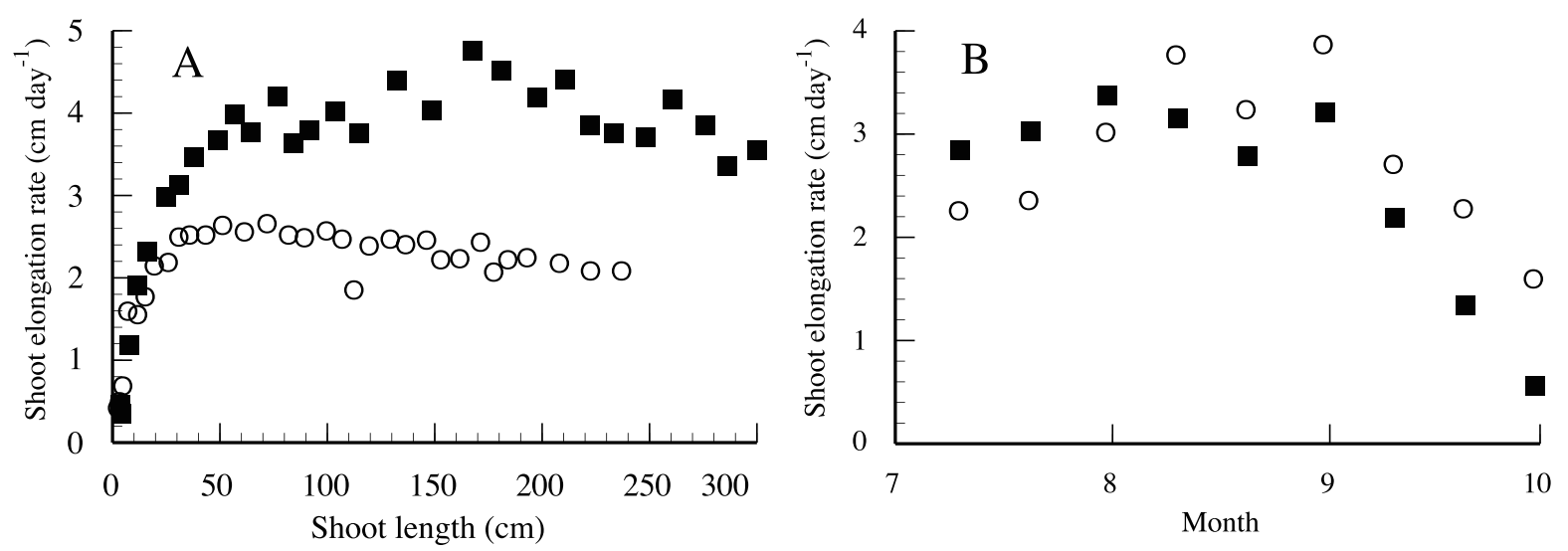

Fig. 3. Relationship between shoot length and shoot elongation rate of mulberry at controlled and field conditions

A: Relationship between shoot length and shoot elongation rate of mulberry at constant temperature and photoperiod (14 hours) conditions.

च: $28^{\circ} \mathrm{C}, \mathrm{O}: 20^{\circ} \mathrm{C}$.

$\mathrm{B}$ : Change in shoot elongation rate of mulberry grown at field conditions.

a: sprouting in April, O: sprouting in June.

length among different temperature conditions was small.

Fig. 3B shows the change in shoot elongation rate of mulberry in field conditions. In this experiment, the mulberries that sprouted in April or June, and the longest shoot in each mulberry plant was measured about every 10 days. After August the shoot elongation rate of mulberry plants that sprouted in April was always slower than plants that sprouted in June. This fact suggests the elongation rate becomes slower with shoot growth after reaching a maximum value.

According to the above results, the shoot elongation rate is described as follows. The sharp increment in the rate after sprouting is represented as a quadratic function of shoot length in PR. The decrement of the rate is described as a linear function of shoot length in GA and the rate decreases after the shoot length reaches an unknown but specific value.

\section{Modeling shoot elongation}

Shoot length data in four prefectures, Miyagi, Fukushima, Ibaraki, and Kagoshima, were used for construction of a mulberry shoot elongation model. Length of the longest shoot in a plant was investigated about every 10 days for three cultivars, 'Ichinose', 'Kairyou-nezumigaeshi' and 'Shin-ichinose', in spring pruning fields and summer pruning fields. Mulberry plants were pruned at the stump base before spring sprouting in the spring pruning fields and at the start of June in the summer pruning fields, respectively. However, in Kagoshima Prefecture, mulberries were pruned at the middle of May in the summer pruning fields. The shoots were sprouting from the stumps after the pruning.

Shoot length $\mathrm{S}$ is determined as follows:

$$
\mathrm{S}(\mathrm{x})=\mathrm{S}(\mathrm{x}-1)+\mathrm{s}
$$

where $S(x)$ is the length of the longest shoot $(\mathrm{cm})$ at the $x$ th day from the initial sprouting day in the spring pruning field and from the first day of measurement in the summer pruning field; and $\mathrm{s}$ is the daily shoot elongation $\left(\mathrm{cm} \mathrm{day}^{-1}\right)$. $\mathrm{s}$ is written as follows:

$$
\mathrm{s}=\mathrm{PR} \cdot \mathrm{GA}
$$

According to the above considerations, PR and GA are written as follows:

$$
\begin{aligned}
\mathrm{PR} & =\mathrm{PR}_{\max } \cdot \mathrm{f}_{1}(\mathrm{~T}) \cdot \mathrm{h}_{1}(\mathrm{~S}) \\
\mathrm{GA}(\mathrm{x}) & =\mathrm{GA}(\mathrm{x}-1) \cdot \mathrm{f}_{2}(\mathrm{~T}) \cdot \mathrm{g}(\mathrm{L}) \cdot \mathrm{h}_{2}(\mathrm{~S})
\end{aligned}
$$

where $\mathrm{PR}_{\max }$ is a parameter and represents the maximum rate of shoot elongation in each prefecture; $\mathrm{T}\left({ }^{\circ} \mathrm{C}\right)$ is the daily average temperature; and L (hour) is the daily photoperiod. Functions are represented by $\mathrm{f}_{1,2}(\mathrm{~T}), \mathrm{g}(\mathrm{L})$ and $h_{1,2}(S) . f_{1,2}(T), g(L)$ and $h_{2}(S)$ are represented as linear functions. A quadric function is used for $h_{1}(S)$. Each function is written as follows:

$$
\begin{aligned}
& \mathrm{f}_{1}(\mathrm{~T})=\mathrm{a}_{1} \cdot\left(\mathrm{T}-\mathrm{b}_{1}\right)+1 \\
& \mathrm{f}_{2}(\mathrm{~T})=\mathrm{a}_{2} \cdot\left(\mathrm{T}-\mathrm{b}_{2}\right)+1 \\
& \mathrm{~g}(\mathrm{~L})=\mathrm{a}_{3} \cdot\left(\mathrm{L}-\mathrm{b}_{3}\right)+1 \\
& \mathrm{~h}_{1}(\mathrm{~S})=-0.25 \cdot \mathrm{a}_{4}{ }^{2} \cdot \mathrm{S}^{2}+\mathrm{a}_{4} \cdot \mathrm{S} \\
& \mathrm{h}_{2}(\mathrm{~S})=\mathrm{a}_{5} \cdot\left(\mathrm{S}-\mathrm{b}_{5}\right)+1
\end{aligned}
$$

where $a_{1-5}$, and $b_{1-5}$ are parameters. For all linear functions, if the functions drop below 0 , they are defined as 0 , and if the functions exceed 1 , the functions are defined as 1. $h_{1}(S)$ is defined as 1 if $\mathrm{S}$ is beyond $2 \cdot a_{4}{ }^{-1}$. To avoid the short photoperiod effect on GA for the early stage of vegetative growth in the spring pruning field, GA is defined as 1 at that period. Since $f_{2}(T)$ represents an interactive 
effect with photoperiod on $\mathrm{GA}, \mathrm{f}_{2}(\mathrm{~T})$ becomes effective in the model after photoperiod $L$ becomes a value below $b_{3}$.

All parameters were estimated by an iterative method, using data from 1997 to 1999 . I used 1 for the first shoot length in the spring pruning field data, and the first measured shoot length in the summer pruning field data. The first value of GA was set at 1 .

\section{Results of shoot elongation estimation}

Fig. 4 shows the relationship between measured and estimated shoot length for 'Ichinose' cultivar in the spring pruning field. The estimated shoot length is similar to the measured values at any time in all years in all prefectures. The estimated shoot length at the last measurement in each year corresponded well with the annual differentiation of measured values (Fig. 5). The model also shows a good prediction for shoot length in 2000 , in which data was not used for parameter determination. These relationships could be seen in the summer pruning field and in other cultivars. Therefore, the constructed model is practical, applicable to a wide area and effective in predicting shoot length at anytime.

Effect of soil condition is reflected in $\mathrm{PR}_{\max }$ in the model. The value of $\mathrm{PR}_{\max }$ in Fukushima was lower than in other prefectures, because soil in Fukushima is sandy and the water condition was worse. Since the values of $\mathrm{PR}_{\max }$ in other prefectures were very similar in each cultivar, and the fertilizer and water conditions seemed to be

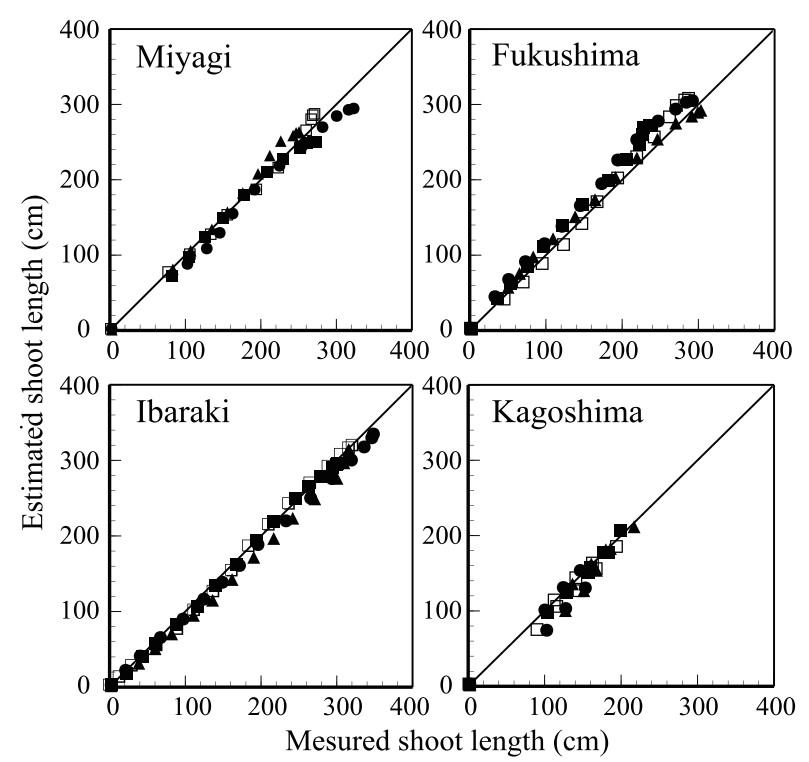

Fig. 4. Relationship between measured and estimated shoot length of "Ichinose" cultivar in spring pruning field

п: 1997, «: 1998, •: 1999, : 2000

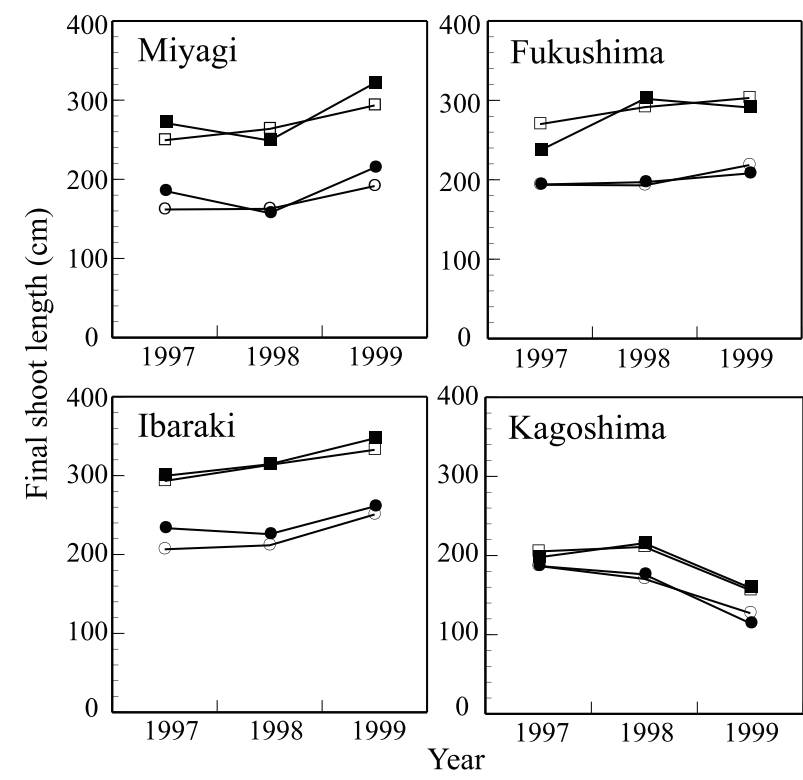

Fig. 5. Relationship between measured and estimated final shoot length of "Ichinose" cultivar from 1997 to 1999

$\bullet-$ : measured values in summer pruning

$\odot$ : estimated values in summer pruning

$\rightarrow-$ : measured values in spring pruning

$\sqsubset:$ estimated values in spring pruning

sufficient in the prefectures, these values seemed to be universal for each cultivar in optimum conditions. Therefore, the model would predict potential shoot elongation growth anywhere with the values of $\mathrm{PR}_{\max }$ in these prefectures except for Fukushima.

\section{References}

1. Fukui, K. (2000) Modeling of shoot elongation and leaf unfolding in potted mulberry. Plant Prod. Sci., 3, 410-416.

2. Fukui, K. (2001) The effects of fluctuating temperature on mulberry sapling growth and dry matter accumulation. $J$. Seric. Sci. Jpn., 70, 31-36.

3. Fukui, K. (2004) Modeling the interactive effect of the photoperiod and temperature on shoot elongation of mulberry. Plant Prod. Sci., 7, 224-229.

4. Fukui, K. (2005) Modeling mulberry shoot elongation and leaf appearance in field conditions. Plant Prod. Sci., 8, 115 121.

5. Fukui, K. (2007) Application of a model for prediction of mulberry shoot elongation to various regions. Nihon Sakumotsu Gakkaikiji (Jpn. J. Crop Sci.), 76, 295-300 [In Japanese with English summary].

6. Kato, T. \& Inoue, F. (1998) A method of the fete case for mulberry yielding of young spring shoots. Saitamaken Sangyou Sikenjou Kenkyu Houkoku (Bull. Saitama Seric. Exp. Stn.), 70, 4-7 [In Japanese].

7. Sugazawa, H. (1968) Studies on the prediction method of the yield of mulberry fields. Sanshi Shikenjou Houkoku 
(Bull. Seric. Exp. Stn.), 22, 329-349 [In Japanese with English summary].

8. Suzuki, M. \& Kanaya, T. (1981) Prediction of shoot elonga- tion in late autumn rearing season. Yamagataken Sangyou Shikenjou Youhou (Bull. Yamagata Seric. Exp. Stn.), 17, 70 73 [In Japanese]. 